\title{
Die Digitalisierung in Studium und Lehre gemeinsam gestalten: Perspektiven aus dem Hochschulforum Digitalisierung
}

\section{Oliver Janoschka, Florian Rampelt, Julius-David Friedrich und Martin Rademacher}

Die Digitalisierung in Studium und Lehre gemeinsam gestalten - dies ist der Anspruch des Hochschulforums Digitalisierung (HFD), der in seiner Vielfalt im vorliegenden Sammelband sichtbar werden soll. Als der Call for Proposals im November 2019 veröffentlicht wurde, ahnte noch niemand, wie bedeutsam dieser Anspruch ab dem Frühjahr 2020 über alle Bildungsbereiche hinweg werden würde.

Das Hochschulforum Digitalisierung beschäftigt sich seit über sechs Jahren mit den Auswirkungen der digitalen Transformation von Bildung, Wissenschaft, Wirtschaft und Gesellschaft auf die Hochschulbildung. Gegründet als gemeinsames Projekt von Stifterverband, Hochschulrektorenkonferenz (HRK) und CHE Centrum für Hochschulentwicklung wird es seit 2014 vom Bundesministerium für Bildung und Forschung gefördert und entwickelt sich kontinuierlich weiter.

Das ,,initiale Anliegen [war dabei,] eine ganzheitliche Sicht auf die Digitalisierung der Lehre an deutschen Hochschulen darzustellen und die damit verbundenen Herausforderungen und Möglichkeiten, Chancen und Risiken zu erfassen und bundesweit zu diskutieren“ (Hochschulforum Digitalisierung 2016, S. 6).

O. Janoschka $(\varangle) \cdot$ F. Rampelt

Stifterverband, Hochschulforum Digitalisierung, Berlin, Deutschland

E-Mail: info@hochschulforumdigitalisierung.de

J.-D. Friedrich

CHE Centrum für Hochschulentwicklung, Hochschulforum Digitalisierung, Gütersloh, Deutschland

M. Rademacher

Hochschulrektorenkonferenz (HRK), Hochschulforum Digitalisierung,

Bonn, Deutschland 


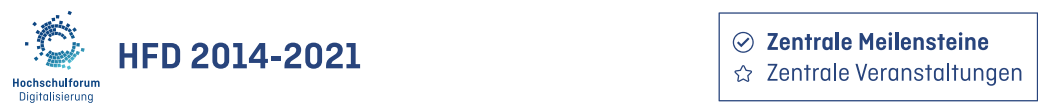

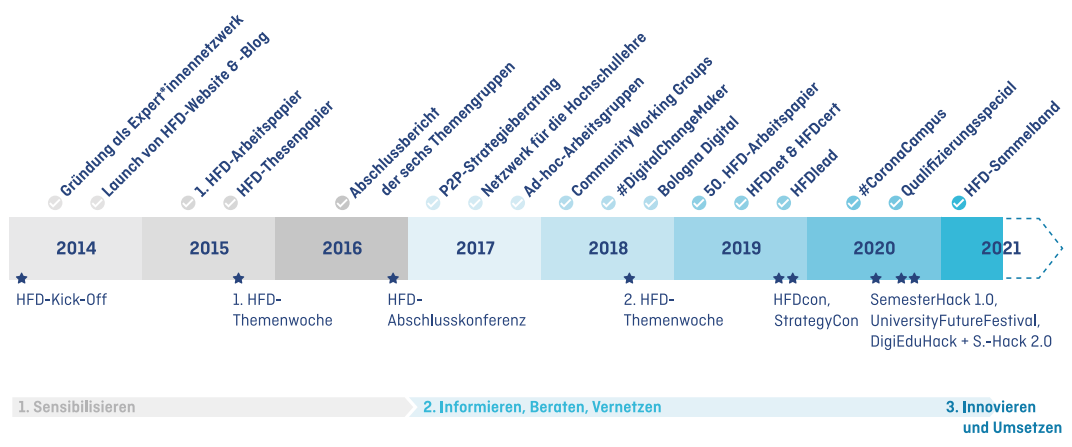

Abb. 1 HFD 2014-2021 (Hochschulforum Digitalisierung, CC BY-SA 4.0)

Das HFD wurde entsprechend mit dem Ziel gegründet, ein institutions- und funktionsübergreifendes Expert*innennetzwerk zu formen, welches in der Lage ist, den Diskurs über die Hochschulbildung im digitalen Zeitalter voranzutreiben. Das HFD hat sich ab 2014 als Thinktank etablieren können, der als zentraler Impulsgeber Akteur*innen aus Hochschulen, Politik, Wirtschaft und Gesellschaft informiert, berät und vernetzt. Im Rahmen seiner Arbeit ist es dem HFD seither gelungen, eine wachsende Anzahl von Hochschulen dabei zu unterstützen, den Prozess der Digitalisierung als Gestaltungschance zu nutzen, sich hochschulübergreifend bei der Bewältigung von bestehenden Herausforderungen zu vernetzen und bei der Entwicklung von neuen Lösungen produktiv zusammenzuarbeiten.

Spätestens in der zweiten Projektphase seit 2017 entwickelte sich das HFD entsprechend sukzessive vom Thinktank zum umsetzungsorientierten Think-\&-DoTank weiter. Neue Instrumente wie die P2P-Strategieberatung oder Community Working Groups machen den Bedarf und das große Interesse der Hochschulen an dem Thema sichtbar und bieten gleichzeitig einen effektiven Unterstützungsprozess an, der nicht nur die Hochschulleitung, sondern im Ansatz die gesamte Institution erreicht. Gleichzeitig wurde der studentischen Perspektive durch die \#DigitalChangeMaker-Initiative (Baumann et al. 2019) ab 2018 eine wichtige Plattform geboten (vgl. Abb. 1 für die zentralen Meilensteine bis 2021). 
Nicht nur die zahlreichen vom HFD bereits erreichten Hochschulen und Individuen, sondern alle akademischen Akteur*innen in Deutschland und Europa waren spätestens im Frühjahr 2020 durch den Ausbruch der Coronapandemie und die damit einhergehende Schließung von Hochschulen dazu aufgefordert, neue Perspektiven einzunehmen. Dies gilt genauso für die strategische Steuerung von Hochschulen wie auch ganz pragmatisch für die operative Umsetzung digitaler und digital gestützter Ansätze für Studium und Lehre (Bils et al. 2020).

Bei allen durch die Digitalisierung angestoßenen Veränderungen und Transformationsprozessen im Hochschulbereich (Gilch et al. 2019) gab es eine Formel, die von Anfang an einen Markenkern der Arbeit des Hochschulforums Digitalisierung darstellte: Die Community, das Gemeinsame, muss im Mittelpunkt stehen, um die deutsche Hochschullandschaft zukunftsfähig aufzustellen. Seit 2014 hat das HFD daher als Forum für Austausch, Vernetzung und Zusammenarbeit der Community eine besondere Priorität eingeräumt. Das HFD begegnet mit seiner Community aus verschiedensten Akteur*innen, die sich mit der Zukunft von Hochschulbildung auseinandersetzen, einem vielfältigen Bedarf nach Unterstützung und Prozessbegleitung und fördert ganz besonders auch das produktive Potenzial der Kollaboration über institutionelle Grenzen hinweg. Über die verschiedenen Community-Formate werden insbesondere die Hochschulen in Deutschland dabei unterstützt, länderübergreifend handlungsfähiger $\mathrm{zu}$ werden. Die Community des Hochschulforums Digitalisierung hat sich gemeinsam mit einer Vielzahl von notwendigen Fragestellungen, kreativen Lösungsansätzen und strategischen Gestaltungsmöglichkeiten der Digitalisierung in Studium und Lehre auseinandergesetzt. Diese Vielfalt, der kreative, gemeinsame Gestaltungsanspruch und auch die hierfür notwendige konzeptionelle Tiefe werden im vorliegenden Sammelband, so hoffen wir, deutlich.

Einen Rahmen hierfür sollen die abschließenden vier Thesen bieten, die sich als Leitgedanken und Erkenntnisse unserer Arbeit beim Hochschulforum Digitalisierung in den vergangenen Jahren verdichtet haben:

\section{Zusammenarbeit ist der Schlüssel zum Erfolg}

Die Digitalisierung in Studium und Lehre kann nur gemeinsam gestaltet werden: „Collaboration is key“! Dies beginnt bereits innerhalb der eigenen Einrichtung, beim fachübergreifenden Miteinander in der täglichen Auseinandersetzung mit guter Lehre. Gleichzeitig können Hochschuleinrichtungen in Zukunft viele Anforderungen der Digitalisierung, ob in Bezug auf technische Lösungen, Lizenzfragen oder die Qualifizierung von Lehrenden und Hochschulmitarbeitenden viel besser institutionenübergreifend angehen. Das Hochschulforum Digitalisierung wiederum ist als bundesweites Forum geprägt 
durch den länderübergreifenden Austausch und eine vertrauensvolle Zusammenarbeit mit den Landesinitiativen, die es in vielen Bundesländern gibt. Für die Zukunft gilt gleichzeitig die europäische Zusammenarbeit als ein Schlüsselelement für kollaborative Maßnahmen im Kontext der digitalen Bildung (Rampelt et al. 2019).

\section{Es braucht Offenheit als Haltung und Strategie}

Die Digitalisierung schafft neue Möglichkeiten der Sichtbarmachung und der gemeinsamen Nutzung von guten Bildungsressourcen sowie von digitalen Technologien. Dabei gilt: Andere haben auch tolle Ideen! Es braucht daher noch mehr Offenheit, Bewährtes zu kopieren und weiterzuentwickeln, ohne dabei die eigene wissenschaftliche Expertise infrage gestellt zu sehen. Die Nutzung von hochwertigen offenen Bildungsressourcen kann mehr Raum für die eigentliche fachliche Aushandlung, den Diskurs und die Betreuung einzelner Studierender bieten. Gleichzeitig schafft Offenheit auch mehr Transparenz in den notwendigen Fragen eines qualitativ hochwertigen Bildungsangebots. Open-Source-Technologien und Interoperabilität können ein Schlüssel für die Gestaltung sicherer und souveräner digitaler Bildungsräume in Europa sein. Diese Offenheit erfordert aber Rechtssicherheit und attraktive Fördermechanismen. Daher braucht es klare Strategien für Open-Source-Systeme und offene Bildungsressourcen.

\section{Partizipation und Empowerment bewirken Bildungserfolg}

Die Bedarfe und Ideen von Studierenden, Lehrenden und Hochschulmitarbeitenden sind zentral für den Erfolg unseres Bildungssystems und der verschiedenen Hochschuleinrichtungen. Dies gilt umso mehr für die langfristige Weiterentwicklung der ad hoc umgesetzten Digitalisierungsmaßnahmen in Zeiten von Corona. Das HFD hat in den vergangenen Jahren enorm von der Vielfalt der beteiligten Statusgruppen profitiert, von Professor*innen über den akademischen Mittelbau bis hin zu Verwaltungs- und Supportmitarbeitenden und den Studierenden. Alle Akteur*innen innerhalb einer Hochschule müssen ernst genommen werden in ihren Anliegen und Unterstützungsangebote geschaffen werden für eine eigenständige Auseinandersetzung mit digitalen Lehr-/Lernszenarien und Technologien. Nur dann kann sich auch der entsprechende Bildungserfolg im Zeitalter der Digitalisierung einstellen. Hierfür sind analoge und digitale Möglichkeiten zum Diskurs, Gesprächsanlässe, konkrete Qualifizierungs- und Mitgestaltungsmöglichkeiten und die Offenheit für neue (oder andernorts bewährte) Ideen erforderlich. Nehmen wir diese ernst!

\section{Innovation erfordert Mut zu neuen, ungewöhnlichen Wegen}

Die digitale Welt ist immer nur so gut, wie wir sie uns gestalten. Um neue Ideen entsprechend auch umzusetzen, braucht es Mut und die Bereitschaft, 
gemeinsam diese neuen Wege zu gehen. Hierfür müssen bestehende Formate, Rollen, Strukturen und Prozesse hinterfragt und weiterentwickelt werden. Die Beiträge im vorliegenden Sammelband geben hier bereits den einen oder anderen kreativen Impuls und zeigen, was möglich ist, wenn man sich gemeinsam auf die digitale Transformation einlässt. Das Hochschulforum Digitalisierung möchte als Mutmacher diesen sicherlich nicht immer einfachen Weg gerne mit allen Akteur*innen der deutschen und europäischen Hochschullandschaft weitergehen. Lassen Sie uns auf dieser Grundlage gemeinsam die Zukunft von Studium und Lehre in Deutschland und in Europa gestalten!

\section{Literatur}

Baumann, J., Böckel, A., Denker, F., Gross, P., Kern, E., Lamprecht, M., Reimann, J., Rensinghoff, B., Sari, Z., Schopf, E., Wächtler, E., Meyer, H., Rampelt, F., \& Röwert, R. (2019). Der Digital Turn aus Studierendenperspektive. Studentisches Thesenpapier zur Digitalisierung in der Hochschulbildung. (Diskussionspapier, 7 (zweite, aktualisierte Version)). Berlin: Hochschulforum Digitalisierung. https://doi.org/10.5281/zenodo.3484574.

Bils, A., Braun, B., Bünemann, T., Scheuring, T., Sutter, C., Meyer, V., Neuner, S., Wagner, B., \& Wistuba, Y. (2020). Corona-Semester 2020 - Ad-hoc-Maßnahmen evaluieren und nachhaltig verankern. (Diskussionspapier, 11). Berlin: Hochschulforum Digitalisierung. https://doi.org/10.5281/zenodo.4247214.

Gilch, H., Beise, A. S., Krempkow, R., Müller, M., Stratmann, F., \& Wannemacher, K. (2019). Digitalisierung der Hochschulen. Ergebnisse einer Schwerpunktstudie für die Expertenkommission Forschung und Innovation. Berlin: EFI.

Hochschulforum Digitalisierung (2016). The Digital Turn - Hochschulbildung im digitalen Zeitalter (Arbeitspapier, 27). Berlin: Hochschulforum Digitalisierung. https://doi.org/10. 5281/zenodo.4282305.

Rampelt, F., Orr, D., \& Knoth, A. (2019). Bologna digital 2020. White paper on digitalisation in the european higher education area. Berlin: Hochschulforum Digitalisierung. https:// doi.org/10.5281/zenodo.3484744. 
Open Access Dieses Kapitel wird unter der Creative Commons Namensnennung 4.0 International Lizenz (http://creativecommons.org/licenses/by/4.0/deed.de) veröffentlicht, welche die Nutzung, Vervielfältigung, Bearbeitung, Verbreitung und Wiedergabe in jeglichem Medium und Format erlaubt, sofern Sie den/die ursprünglichen Autor(en) und die Quelle ordnungsgemäß nennen, einen Link zur Creative Commons Lizenz beifügen und angeben, ob Änderungen vorgenommen wurden.

Die in diesem Kapitel enthaltenen Bilder und sonstiges Drittmaterial unterliegen ebenfalls der genannten Creative Commons Lizenz, sofern sich aus der Abbildungslegende nichts anderes ergibt. Sofern das betreffende Material nicht unter der genannten Creative Commons Lizenz steht und die betreffende Handlung nicht nach gesetzlichen Vorschriften erlaubt ist, ist für die oben aufgeführten Weiterverwendungen des Materials die Einwilligung des jeweiligen Rechteinhabers einzuholen.

(c) () 